\title{
The Prevalence and Severity of Gallstones in Sickle Cell Disease in Kuwait
}

\author{
Hassan A. Al-Jafar ${ }^{1 *}$, Hussein A. Hayati ${ }^{2}$, Hanaa AlKhawari ${ }^{3}$, Fatmah S. AlThallab4, \\ Masouma Al-Ali ${ }^{5}$
}

${ }^{1}$ Hematology Department, Amiri Hospital, Ministry of Health, Kuwait City, Kuwait

${ }^{2}$ Hepatobiliary Surgery Department, Amiri Hospital, Ministry of Health, Kuwait City, Kuwait

${ }^{3}$ Radiology Department, Amiri Hospital, Ministry of Health, Kuwait City, Kuwait

${ }^{4}$ National Center for Kuwait Health Information, Ministry of Health, Kuwait City, Kuwait

${ }^{5}$ Liver \& Gastroenterology Department, Amiri Hospital, Ministry of Health, Kuwait City, Kuwait

Email: *haj400004@gmail.com, *cbc9@hotmailcom, hhayati67@hotmail.ca, halkhawari@moh.gov.kw,

al_thallab2005@yahooo.com, manayer1@hotmail.com

How to cite this paper: Al-Jafar, H.A., Hayati, H.A., AlKhawari, H., AlThallab, F.S. and Al-Ali, M. (2020) The Prevalence and Severity of Gallstones in Sickle Cell Disease in Kuwait. Open Journal of Epidemiology, 10, 346-354. https://doi.org/10.4236/ojepi.2020.104028

Received: July 17, 2020

Accepted: August 31, 2020

Published: September 3, 2020

Copyright ( 2020 by author(s) and Scientific Research Publishing Inc. This work is licensed under the Creative Commons Attribution International License (CC BY 4.0).

http://creativecommons.org/licenses/by/4.0/ (c) (i) Open Access

\begin{abstract}
Introduction: Sickle cell disease (SCD) is a genetic disease affecting hemoglobin development. Complications may occur in all organs due to sickle cell hemoglobin. Gallstones may develop as the complication of the biliary system in SCD. Aim: To calculate the prevalence and severity of the biliary system complications in SCD. Method: A total of 220 patients with homozygous SCD were recruited. The prevalence of gallstones was estimated, and the severity of the biliary system complications was classified according to the condition of the gallstones; it was classified as grade 0 when no gallstones were detected, grade 1 when gallstones were present only in the gallbladder, grade 2 when gallstones were present in both the gallbladder and the common bile duct, and grade 3 when the patient had cholecystectomy due to gallstones. Results: The overall prevalence of gallstones and cholecystectomy was 51\%; it was $22 \%$ in females and $29 \%$ in males. The prevalence of the severity of grade 0 was $49 \%$, grade 1 was $14 \%$, and grade 3 was $37 \%$. Grade 2 prevalence was not calculated because this study was based on abdominal ultrasound only. Conclusion: The prevalence of gallstones in SCD is much higher than in the normal population, and more in males than in females. It begins at an early age during childhood due to several underlying etiological factors related to SCD. This study provided a simple grading of severity for the biliary system based on the gallbladder stone complication. The severity calculation in the biliary system is a part of the assessment of the severity in other systems in this multisystem chronic disorder.
\end{abstract}




\section{Keywords}

Sickle Cell Disease, Gallbladder, Stone, Severity

\section{Introduction}

Sickle cell disease (SCD) is caused by a gene affecting hemoglobin development. Complications arise due to changes in the shape of the erythrocytes during deoxygenation, secondary to the polymerization of the abnormal sickle hemoglobin (HbS). Sickled erythrocytes cause vaso-occlusion along with abnormal endothelial interactions, leading to repeated ischemia, inflammation, and endothelial dysfunction. All organs may be affected. In this chronic congenital disorder, the frequency of organ dysfunctions increases with age [1]. Gall bladder stones (GBS), also known as cholelithiasis, are a common complication of SCD and its variants [2]. The sickle red blood cells have a shorter life span than the normal ones. Chronic hemolysis leads to the continuous production of bilirubin, which gets conjugated in the liver and excreted in the feces as urobilinogen, in large quantities, forming calcium bilirubinate gallstones. Cholelithiasis can be detected even in children under five years of age, but is more common in adolescents and adults with SCD. Due to its potential risk, early diagnosis is important [3]. GBS could also be silent, but symptoms and signs could present in the form of colicky pain in the upper-right side of the abdomen, nausea, vomiting, pain between shoulder blades or below the right shoulder, itching, and jaundice [4]. Common bile duct stones (CBDS), also known as choledocholithiasis, are the presence of gallstones in the common bile duct that can migrate and block the common bile duct [5]. Clinical complications include abdominal pain, jaundice, nausea, vomiting, elevated levels of bilirubin, or liver enzymes [6].

There is no agreement on the disease severity worldwide because of the variable nature of the disease. It could be degenerative, neoplastic, infectious, vascular, autoimmune, or due to several other causes. Thus, assessment of severity for all these disorders on a single scale is not possible. Disease severity assessment corresponds to understanding the degree of damage in order to aid in management. The severity of the disease should be easy for the patient to understand. Moreover, the criteria for severity assessment must have validation, should be applied by the treating doctors, and must be accepted by the experts of the specialty [7].

This study aimed to calculate both the prevalence and severity of GBS in SCD, and to establish uncomplicated criteria for severity assessment that can be easily applied by the hematologists, surgeons, and other health care-givers.

\section{Methods}

GBS is quite a common complication in SCD. We used GBS as an indicator for the biliary system complication in SCD, because of its easy detection by the sim- 
ple abdominal ultrasound ( $\mathrm{ABU}$ ).

This study is part of a project for estimating the severity in each system in SCD to provide a total severity status of all body organs for each patient, as such patients have great variations in the severity of their SCD in the different systems in the body.

\subsection{Study Area and Study Population}

The study population comprised all cases of SCD in children and adults recruited from the Amiri hospital SCD specialized outpatient clinics and from other hospitals, including Mubark Al-Kabeer, Adan, Sabah, Farwania Hospitals, and 12 family medicine polyclinics in the capital health region from the year 2016 to 2019. The study population represented approximately a quarter of the total SCD patients in Kuwait. Inclusion criterion was all SCD patients in our cohort who underwent ABU. Exclusion criteria were cholecystectomy or gallstones due to factors other than SCD.

\subsection{Data Collection and Analysis}

\section{Statistical Analysis}

The data collected included age, sex, and clinical signs and symptoms. The data were validated manually and were analyzed to determine the confidence interval for a population proportion. The confidence interval for population proportion was used to get $\mathrm{p}$-value at 0.05 significant level. The prevalence of GBS in SCD was estimated, and the severity of the biliary system disease related to GBS was classified into four grades: grade 0 , normal; grade 1, mild; grade 2, moderate; and grade 3, severe. Grade 0 was considered when abdominal ultrasound was normal. Grade 1 (mild) when GBS were found only in either gallbladder or common bile duct. Grade 2 (moderate) when GBS were found in both the gallbladder and the bile duct. Grade 3 (severe) in cases of cholecystectomy related to SCD due to GBS [Figure 1]. Grade 2 was not estimated in this study, as no cholecystography, magnetic resonance cholangiopancreatography (MRCP), or endoscopic retrograde cholangiopancreatography (ERCP) was performed as a routine diagnostic test. The prevalence calculated in each group of severity was related to SCD prevalence in the capital area in Kuwait.

\subsection{Ethical Considerations}

Ethical approval was obtained from the combined Ethics Committee of the Kuwait Faculty of Medicine and The Ministry of Health in the state of Kuwait. This study was part of the "Sickle Cell Disease Classification According to the Systemic Complications" project, approved and partially funded by the Kuwait Foundation for the Advancement of Sciences (KFAS).

\section{Results}

This prospective study was conducted on 220 SCD patients comprising $101 \mathrm{fe}$ - 
males and 119 males in the Capital Health Area in the state of Kuwait. The total population related to this area in 2018 was about 584,313 with 326,358 males and 257,955 females [8]. The results revealed prevalence of normal GB as $49 \%$, GB Stones as $14 \%$, and cholecystectomy as $37 \%$ [Figure 2]. In relation to gender, the prevalence of grade 0 in females was $24 \%$ and in males was $25 \%$; grade 1 in females was $4 \%$ and in males was $10 \%$; and grade 3 in females was $18 \%$ and in males was 19\% [Table 1], [Figure 3]. More stones indicated more severity, and the cholecystectomy indicated organ failure, which is the highest grade of severity. In relation to age group, the grade 0 prevalence was $16 \%$ in children and $84 \%$ in adults; grade 1 was with $2 \%$ in children and $12 \%$ in adults; and grade 3 was $0.005 \%$ in children and $37 \%$ in adults [Table 2] [Figure 4]. The prevalence was also affected by the number of patients attending the study, while the severity was affected by many underlying causes, such as the genotype variants of SCD, patient compliance to treatment, and individual underlying factors for each patient. With the periodic follow up, the patients' severity may increase from lower to higher grades based on the $\mathrm{ABU}$ results.

\section{Discussion}

SCD is a chronic disorder with multisystem complications. Each patient has a different degree of complications in each organ system, and every SCD case is different in its severity. A classification system was invented to assess the overall severity of each case of SCD, and to assess the clinical severity, as many SCD complications can be hidden. This study is part of a project on assessing the severity of SCD in each system to reach to an SCD classification of severity in each SCD patient [9]. The assessment of prevalence is beneficial for the general public

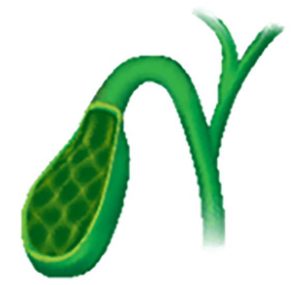

Grade 0: Normal gallbladder

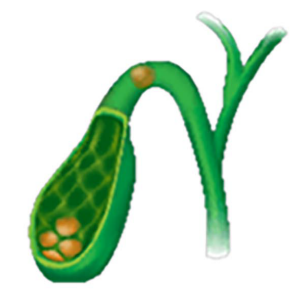

Grade 2: GBS and bile duct stones

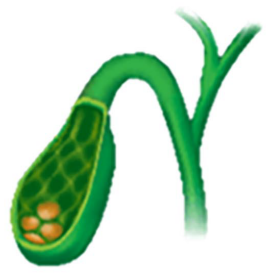

Grade 1: GBS or bile duct stones

Figure 1. Severity classification of gallstone (GBS) complication. 


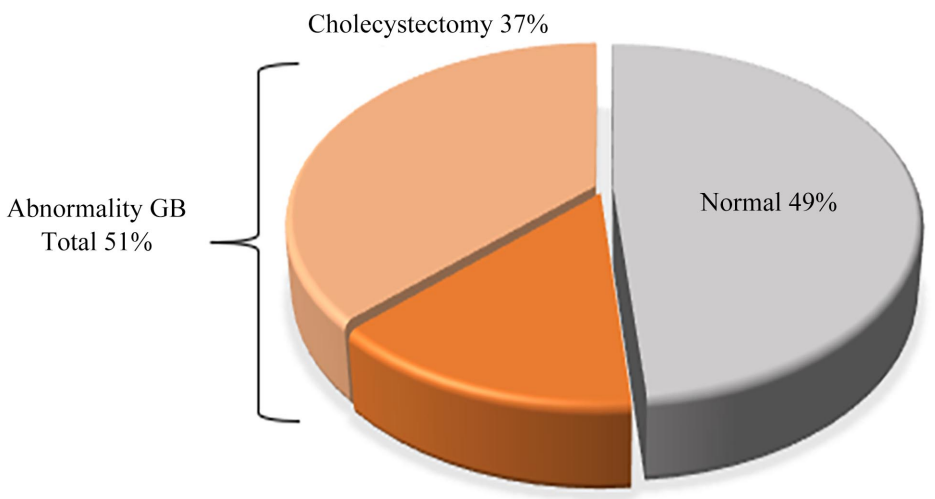

GB Stones 14\%

Figure 2. Prevalence of Gallstone Disease in Sickle cell disease.

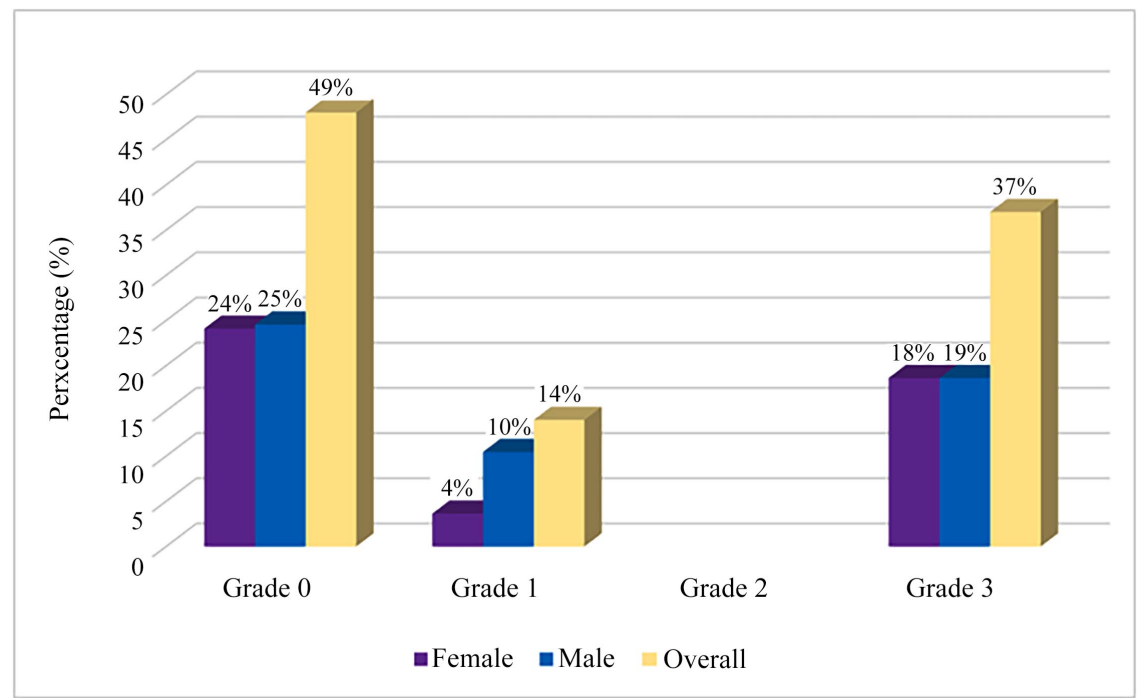

Figure 3. Grading distribution of gallstones in SCD severity in Gender group.

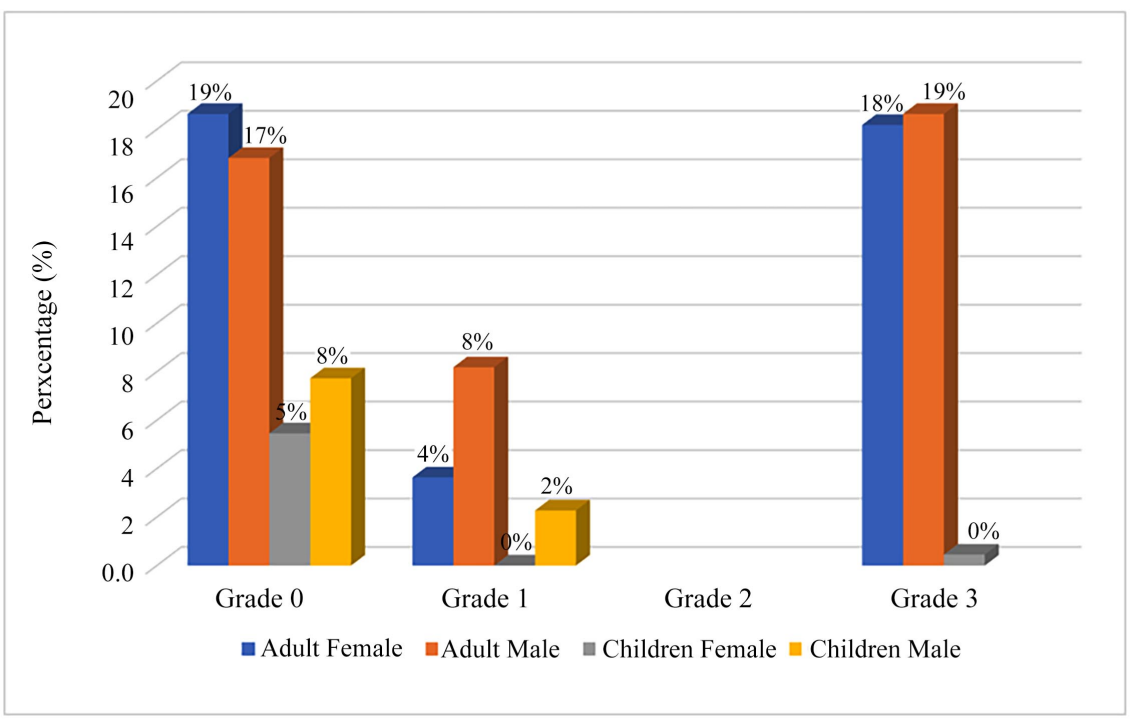

Figure 4. Grading distribution of SCD severity in the age group. 
Table 1. The prevalence of gall stones according to the gender in SCD patients.

\begin{tabular}{cccccc}
\hline Cases & Severity grade & Female & Male & Total & P VALUE \\
\hline Total & & 101 & 119 & 220 & \\
Normal & Normal $=0$ & $24 \%(53)$ & $25 \%(54)$ & $49 \%(107)$ & 0.0023 \\
GB Stones & Mild $=1$ & $4 \%(8)$ & $10 \%(23)$ & $14 \%(31)$ & 0.0062 \\
Cholecystectomy & Sever $=3$ & $18 \%(40)$ & $19 \%(42)$ & $37 \%(82)$ & 0.0025 \\
\hline
\end{tabular}

Table 2. The prevalence of gallstones according to the age in SCD patients.

\begin{tabular}{|c|c|c|c|c|c|c|c|c|}
\hline Cases & Severity grade & $\mathrm{CF}$ & $\mathrm{CM}$ & P VALUE & $\mathrm{AF}$ & $\mathrm{AM}$ & Total & P VALUE \\
\hline Total & & 13 & 22 & & 89 & 96 & & \\
\hline Normal & Normal $=0$ & $5 \%(12)$ & $8 \%(17)$ & 0.0083 & $19 \%(41)$ & $17 \%(37)$ & $49 \%(107)$ & 0.0032 \\
\hline GB Stones & Mild = 1 & & $2 \%(5)$ & & $4 \%(8)$ & $8 \%(18)$ & $14 \%(31)$ & 0.0082 \\
\hline Cholecystectomy & Sever $=3$ & $0.005 \%(1)$ & & & $18 \%(40)$ & $19 \%(41)$ & $37 \%(82)$ & 0.0128 \\
\hline Total & & & & & & & & \\
\hline
\end{tabular}

Note: children males (CM), children females (CF), Adults male (AM), Adults female (AF).

health policy for the medical authorities, and estimating the severity has multiple advantages for each patient's personal health care. This study calculated both the prevalence and the degree of severity for GBS status in SCD. In non-SCD individuals, GBS occurs as a result of complex interactions of genetic, environmental, metabolic, and other risk elements. Factors for increased risk of gallstones include the female gender; age of more than 40 years; obesity; being pregnant; consuming diet rich in fats, high-cholesterol, and low-fibers; taking medications containing estrogen, oral contraceptives, hormonal therapy; or having liver disease [10]. Several risk factors remain conflicting due to differences in epidemiological approaches [11]. In the literature, the prevalence of cholelithiasis in the normal population is $9 \%$ in females and $6 \%$ in males, with about $80 \%$ asymptomatic cases, and the presence of choledocholithiasis in $4.4 \%-18.8 \%$ cases [12]. In our cohort study of 220 patients with homozygous SCD, aged $2-82$ years, the prevalence of gallbladder SCD is shown in Table 1 . This prevalence was consistent with the prevalence of GBS in SCD investigated with ABU in UK, where in 95 SCD patients aged 10 - 65 years, 58\% (55) patients had GBS or underwent cholecystectomy [13]. In our results, the gender group prevalence was $51 \%$, with GBS in $14 \%$ and cholecystectomy secondary to SCD in $37 \%$ cases. The female prevalence of GBS was less than the male prevalence, which is contrary to the usual prevalence in the non-SCD population. This finding is important as it indicates that GBS in SCD is a prominent finding in both the genders, and that other factors might also have a role in this unusual result in the different variant types of homozygous SCD [14].

In this study, the number of complications increased with age, which was expected as SCD is a chronic disorder [15]. Moreover, the GBS complications were found in $16 \%$ of the children and $84 \%$ of the adults. This confirmed the increase 
of GBS with age in this chronic disorder [Table 2]. Age is a major risk factor for the development of GBS in SCD as observed in other studies [10]. The extremely high prevalence of GBS in SCD indicates that it has unique and many etiological factors causing GBS due to chronic hemolytic anemia, liver sickle red blood cell sequestration, hepatic infarction, and several other factors [16]. This finding highlights the importance of $\mathrm{ABU}$ investigation for detecting early biliary tract complications.

To overcome gallbladder and other SCD complications, hydroxycarbamide treatment may be used from early childhood with appreciable effect [17].

The GBS severity in our cohort was characterized using a simple and reliable method and the findings were grade 0 in 107 patients (49\%) and grade 1 in 31 patients (14\%); grade 2 was not analyzed as this study was only based on $\mathrm{ABU}$. In cases where common bile duct stones were suspected, MRCP or ERCP was performed to detect the existence of CBD stones, with grade 3 severity denoting an organ failure. In this study, the cholecystectomy due to SCD gallbladder stones was assigned as an organ failure. The existence of cholecystectomy in children indicated the severity of GBS in this group. The GBS was detected in patients aged $4-72$ years and cholecystectomy in those aged $11-82$ years. The annual $\mathrm{ABU}$ assessment for the biliary system is important to rule out GBS and to avoid its complications in SCD.

The severity of GBS provides each patient with the required medical care according to their degree of severity. In the literature, there is no agreement regarding the precise definition of the severity. Severity is defined as the total effect of the disease on the organ function. Any severity scale should have validity, and acceptance of the grades as being clinically credible within each organ system from the experts in the field [18]. ABU is an essential first-line investigation in suspected cases of GBS in SCD. It is a highly sensitive, accurate, and cheap imaging modality. It is a non-invasive technique with $90 \%-95 \%$ accuracy in detecting gallstones and gallbladder sludge [19]. The formation of gallstones often takes months or years, depending on the size and quantity of the stones. Thus, according to an approved protocol from the medical authority, follow up in cases of SCD is mandatory with periodic investigations [20].

\subsection{Limitations}

GBS is a reliable method to estimate the biliary tract severity by ABU. Although some authors may include acute and chronic cholecystitis in the severity grading of the biliary system, we could not find validity in using acute or chronic intermittent complications that are not usually detectable by $\mathrm{ABU}$ or other investigations, though some patients might be affected by them. Other limitation was that the total number of male patients was slightly more than the number of females.

\subsection{Conclusion}

This study was conducted to create a severity grading for the biliary tract system. 
The grading of the severity is part of the classification of SCD severity in each patient. The severity of the biliary tract system is based on $\mathrm{ABU}$ findings. The severity is graded from $0-3$. The prevalence of biliary tract gall stones was also calculated in this study to assess the burden of gallbladder stones. The prevalence was higher in SCD patients with an unusual existence in young children and it was more in males. The severity calculation was designed to be simple for application by physicians treating SCD patients with multisystem complications. The severity assessment by $\mathrm{ABU}$ was found reliable with high validity. This study also shows the importance of a periodic follow-up and the annual ABU to provide the necessary awareness of the complications in SCD patients, and to treat them at the right time.

\section{Acknowledgements}

This research work is done under the "Sickle Cell Disease Classification According to the Systemic Complications" which has Ministry of Health ethical approval number DVR/906, and funded "Partially" by Kuwait Foundation for the advancement of science (KFAS), under code: P116-13MM-01.

\section{Conflicts of Interest}

The authors have no conflict of interest in this study.

\section{References}

[1] Ware, R.E., De Montalembert, M., Tshilolo, L. and Abboud, M.R. (2017) Sickle Cell Disease. The Lancet, 390, 311-323. https://doi.org/10.1016/S0140-6736(17)30193-9

[2] Johnson, C. (2001) Gall Bladder and Liver Disorders in Sickle Cell Disease: A Critical Review.

[3] Gumiero, A.P., Bellomo-Brandão, M.A. and Costa-Pinto, E.A. (2008) Gallstones in Children with Sickle Cell Disease Followed up at a Brazilian Hematology Center. Arq Gastroenterol., 45, 313-318. https://doi.org/10.1590/S0004-28032008000400010

[4] Wenckert, A. and Robertson, B. (1966) The Natural Course of Gallstone Disease. Gastroenterology, 50, 376-381. https://doi.org/10.1016/S0016-5085(66)80078-1

[5] Martins, R.A., Soares, R.S., Vito, F.B., et al. (2017) Cholelithiasis and Its Complications in Sickle Cell Disease in a University Hospital. Revista Brasileira de Hematologia e Hemoterapia, 39, 28-31. https://doi.org/10.1016/j.bjhh.2016.09.009

[6] Almadi, M.A., Barkun, J.S. and Barkun, A.N. (2012) Management of Suspected Stones in the Common Bile Duct. CMAJ, 184, 884-892. https://doi.org/10.1503/cmaj.110896

[7] Jensen, P.M. and De Fine Licht, H.H. (2016) Predicting Global Variation in Infectious Disease Severity: A Bottom-Up Approach. Evolution, Medicine, and Public Health, 2016, 85-94. https://doi.org/10.1093/emph/eow005

[8] The Public Authority for Civil Information, Kuwait. https://www.citypopulation.de/en/kuwait/admin

[9] Al-Jafar, H. (2017) Sickle Cell Disease Clinical Classification. Blood, 130, 4771.

[10] Debray, D., Franchi-Abella, S., Irtan, S. and Girard, M. (2012) Cholelithiasis in Infants, Children and Adolescent. La Presse Médicale, 41, 466-473. 
https://doi.org/10.1016/j.lpm.2011.09.018

[11] McNicoll, C.F., Pastorino, A., Farooq, U., et al. (2020) Choledocholithiasis. StatPearls Publishing, Treasure Island.

[12] Eun-Hyung, Y. and Soo-Youn, L. (2009) The Prevalence and Risk Factors for Gallstone Disease. Clinical Chemistry and Laboratory Medicine (CCLM), 47, 795-807. https://doi.org/10.1515/CCLM.2009.194

[13] Bond, L.R., Hatty, S.R., Horn, M.E., Dick, M., Meire, H.B. and Bellingham, A.J. (1987) Gall Stones in Sickle Cell Disease in the United Kingdom. British Medical Journal, 295, 234-236. https://doi.org/10.1136/bmj.295.6592.234

[14] Novacek, G. (2006) Gender and Gallstone Disease. Wiener Medizinische Wochenschrift, 156, 527-533. https://doi.org/10.1007/s10354-006-0346-X

[15] Martins, R.A., Soares, R.S., Vito, F.B., et al. (2017) Cholelithiasis and Its Complications in Sickle Cell Disease in a University Hospital. Revista Brasileira de Hematologia e Hemoterapia, 39, 28-31.

[16] Al-Jafar, H., Alkhaldi, J.A. and Termos, S. (2020) Etiology Based Sickle Cell Disease Hepatopathy. Open Journal of Gastroenterology, 10, 187-201.

[17] McGann, P.T. and Ware, R.E. (2015) Hydroxyurea Therapy for Sickle Cell Anemia. Expert Opinion on Drug Safety, 14, 1749-1758. https://doi.org/10.1517/14740338.2015.1088827

[18] Medsger Jr., T.A., Bombardieri, S., László, C., Scorza, R., Della Rossa, A. and Bencivelli, W. (2003) Assessment of Disease Severity and Prognosis in SSc. Clinical and Experimental Rheumatology, 21, S42-S46.

[19] Negrão de Figueiredo, G., Mueller-Peltzer, K., Zengel, P., Armbruster, M., Rübenthaler, J. and Clevert, D.A. (2019) Contrast-Enhanced Ultrasound (CEUS) and Gallbladder Diseases-A Retrospective Mono-Center Analysis of Imaging Findings with Histopathological Correlation. Clinical Hemorheology and Microcirculation, 71, 151-158. https://doi.org/10.3233/CH-189405

[20] Sackman, M. (2018) The Informed Patient: What You Should Know about Gallstone Treatment. 15th Revised Edition, Falk Foundation, Pittsburgh. http://www.drfalkpharma.com/uploads/tx tocfpshoperw/U85e 15-5-18.pdf 